\title{
O Estresse no ambiente Hospitalar
}

\author{
Stress in the Hospital Environment.
}

El estrés en el ambiente hospitalario

\section{Danieli Silva Carvalho $^{1 *}$, Eduardo Tavares Lima Trajano², Sebastião Jorge da Cunha Gonçalves ${ }^{3}$}

Como citar esse artigo. Carvalho, DS; Trajano, ETL; Gonçalves, SJC. O Estresse no ambiente Hospitalar. Revista Pró-UniverSUS. 2019 Jan./Jun.; 10 (1): $76-80$

\section{Resumo}

Este estudo objetiva evidenciar na literatura científica, as contribuições do enfermeiro no autocuidado ao paciente estomizado. Por se tratar de um momento de mudanças na imagem corporal, alterações complexas no cotidiano e vida social, com necessidades de enfrentamento, em face a sentimentos como medo, depressão, e ajuste familiar, torna-se mais relevante a atuação do enfermeiro. Trata-se de um estudo de revisão integrativa e foi desenvolvida através da identificação do tema e seleção da hipótese ou questão norteadora da pesquisa, estabelecimento dos critérios de inclusão e exclusão da pesquisa, definição das informações a serem extraídas dos estudos selecionados, avaliação dos estudos incluídos na revisão integrativa, interpretação dos resultados e síntese do conhecimento. Cada paciente tem sua particularidade, precisa ser visto de forma singular, com visão holística, e o enfermeiro, fundamentada em orientações científicas, e na busca de qualidade de vida, é capaz de ajudar o paciente em sua reabiltação, adaptação, recuperação de autoestima e capacidade produtiva.

Palavras-chave: Enfermagem, Estomia, Autocuidado.

\begin{abstract}
Este estudio objetiva evidenciar en la literatura científica, las contribuciones del enfermero en el autocuidado al paciente estomizado. Por tratarse de un momento de cambios en la imagen corporal, alteraciones complejas en el cotidiano y la vida social, con necesidades de enfrentamiento, frente a sentimientos como miedo, depresión, y ajuste familiar, se vuelve más relevante la actuación del enfermero. Se trata de un estudio de revisión integrativa y fue desarrollada a través de la identificación del tema y selección de la hipótesis o cuestión orientadora de la investigación, establecimiento de los criterios de inclusión y exclusión de la investigación, definición de las informaciones a ser extraídas de los estudios seleccionados, evaluación de los estudios seleccionados incluidos en la revisión integrativa, interpretación de los resultados y síntesis del conocimiento. Cada paciente tiene su particularidad, necesita ser visto de forma singular, con visión holística, y el enfermero, fundamentado en orientaciones científicas, y en la búsqueda de calidad de vida, es capaz de ayudar al paciente en su rehabilitación, adaptación, recuperación de autoestima y recuperación capacidad productiva.
\end{abstract}

Keywords: Enfermería, ostomía, Autocuidado.

\section{Resumen}

Este estudio objetiva evidenciar en la literatura científica, las contribuciones del enfermero en el autocuidado al paciente estomizado. Por tratarse de un momento de cambios en la imagen corporal, alteraciones complejas en el cotidiano y la vida social, con necesidades de enfrentamiento, frente a sentimientos como miedo, depresión, y ajuste familiar, se vuelve más relevante la actuación del enfermero. Se trata de un estudio de revisión integrativa y fue desarrollada a través de la identificación del tema y selección de la hipótesis o cuestión orientadora de la investigación, establecimiento de los criterios de inclusión y exclusión de la investigación, definición de las informaciones a ser extraídas de los estudios seleccionados, evaluación de los estudios seleccionados incluidos en la revisión integrativa, interpretación de los resultados y síntesis del conocimiento. Cada paciente tiene su particularidad, necesita ser visto de forma singular, con visión holística, y el enfermero, fundamentado en orientaciones científicas, y en la búsqueda de calidad de vida, es capaz de ayudar al paciente en su rehabilitación, adaptación, recuperación de autoestima y recuperación capacidad productiva.

Palabras clave: Enfermería, ostomía, Autocuidado.

\section{Afiliação dos autores:}

1Acadêmico do Curso de Enfermagem, Universidade de Vassouras. RJ, Brasil. Email: carvalhodanieli1987@outlook.com ORCID: https://orcid.org/0000-0002-1774-3586

2 Doutor. Professor do Curso de Enfermagem. Cooordenado do Mestrado Profissional em Ciências Aplicadas em Saúde. Universidade de Vassouras. RJ, Brasil.

Email:eduardolimatrajano@hotmail.comORCID: https://orcid.org/0000-0001-7809-7138

3Mestre. Professor do Curso de Enfermagem, Universidade de Vassouras. RJ, Brasil. Email: sjcunha@uol.com.br ORCID: https://orcid.org/0000-0002-4228-4641 


\section{Introdução}

O estresse é considerado um dos principais fatores responsáveis por alterações do estado de saúde da população mundial, manifes $\neg$ tando-se por meio de alterações fisiológicas, cognitivas e comportamentais, po $\neg$ dendo, assim, levar ao surgimento de doenças e até à morte. A compreensão do estresse como um processo psicofisiológico do organismo é fundamental, pois permite diagnosticar as respostas desencadeadas pela maneira como os es $\neg$ tímulos são processados.

$\mathrm{O}$ estresse quanto a adaptação existe em níveis diferentes de um sistema, é possível estudar essas reações nos níveis celular, tecidual e orgânico. Os biólogos estão preocupados principalmente com os componentes subcelulares ou com os subsistemas do corpo como um todo. Os cientistas comportamentais, incluindo os pesquisadores da enfermagem, estudam o estresse e a adaptação em indivíduos, familiares, grupos e sociedades; eles enfocam o modo como às características organizacionais deum grupo semodificam para atender as necessidades do ambiente social e físico no qual o grupo está inserido. Em qualquer sistema, os objetivos desejados da adaptação são a sobrevivência, o crescimento e a reprodução. ${ }^{1: 78}$

Nos dias de hoje, procurar e ser atendido numa instituição hospitalaré algo rotineiro e frequente. Esperase que o hospital tenha uma equipe interdisciplinar nas várias especialidades, que disponha de recursos técnicos avançados e atenda de forma rápida e ininterrupta..$^{2: 15}$ No entanto, o atendimento em uma instituição hospitalar nem sempre ocorre dessa maneira, gerando estresse por parte dos pacientes e familiares.

Relacionado à patologia está à reação do organismo e, comumente, a gravidade da enfermidade deve-se primeiramente a violência do corpo e suas defesas naturais contra infecções e outras doenças. ${ }^{3}$

O presente trabalho tem por objetivo expor o tema estresse em suas definições e expor o levantamento de dados em pesquisa através de artigos científicos publicados.

O trabalho tem a pretensão de estudar o tema Estresse no ambiente hospitalar na promoção da saúde dos pacientes internados e equipe multiprofissional. Dando enfoque aos manejos desenvolvidos pelos profissionais da Enfermagem.

\section{Metodologia}

Trata-se de uma pesquisa bibliográfica de abordagem qualitativa e caráter descritivo. Cabe ressaltar que a pesquisa bibliográfica que é desenvolvida com auxílio de material já elaborado, constituído principalmente de livros e artigos científicos. ${ }^{4}$

Os dados foram coletados em Base de Dados Virtuais. Para tal utilizou-se a Biblioteca Virtual de Saúde (BVS), na seguinte Base de informação: Literatura Latino-Americana e do Caribe em Ciências da Saúde (LILACS), Literatura Internacional em Ciência da Saúde (MEDLINE), Base de Dados de Enfermagem (BDENF) e pelo endereço eletrônico scholar.google. com.br, no período de Março à Outubro de 2018.

Tabela 1. Estágios do estresse

\begin{tabular}{|c|c|}
\hline Estagio & Caracterísitcas \\
\hline $\begin{array}{l}1^{\circ} \text {. Estágio: } \\
\text { ALARME }\end{array}$ & $\begin{array}{l}\text { O organismo tem uma excitação de agressão ou de fuga ao estressor, que pode ser } \\
\text { entendida como um comportamento de adaptação. Este estágio é caracterizado por alguns } \\
\text { sintomas como dor de cabeça, esgotamento, extremidades frias, pressão no peito, taquicardia, } \\
\text { tensão crônica, dentre outros. }\end{array}$ \\
\hline $\begin{array}{l}2^{\circ} \text {. Estágio: } \\
\text { RESISTENCIA }\end{array}$ & $\begin{array}{l}\text { Havendo persistência da fase de alerta, o organismo altera seus parâmetros de } \\
\text { normalidade e concentra a reação interna num determinado órgão. E o momento onde o } \\
\text { individuo procura adaptar-se ao estresse, havendo uma liberação exagerada de adrenalina. } \\
\text { Aqui se manifestam sintomas psicossociais: alteração do apetite, ansiedade, impotencia } \\
\text { sexual, isolamento social, medo e outros. }\end{array}$ \\
\hline $\begin{array}{l}3^{\circ} \text {. Estágio: } \\
\text { EXAUSTÄO }\end{array}$ & $\begin{array}{l}\text { O individuo é incapaz de manter o nível de resistência e o organismo encontra-se } \\
\text { esgotado pelo excesso de atividades e pelo alto consumo de energia, podendo ocorrer infarto, } \\
\text { hipertensão arterial sistêmica, úlceras e outras. }\end{array}$ \\
\hline
\end{tabular}

Fonte: Selye (1956) apud Camelo e Angerami (2004, p.15). 
A estratégia de pesquisa utilizada para busca dos artigos foi: Estresse AND Enfermagem AND Hospitalização.

\section{Resultados}

Após a associação de todos os descritores nas bases pesquisadas foram encontrados 10 artigos.

Pode ser observado no gráfico 1 (um) que o maior número de artigos publicados fazendo referencia ao estresse no âmbito hospitalar é em 2009 e 2017 com quatro publicações, sendo dois artigos para cada ano. Justifica-se essa quantidade, pelo fato consequentemente de interesse pessoal dos autores para o ano publicado, sabendo que esse assunto em qualquer momento do tempo será sempre um assunto atual.
Analisando o gráfico de número 02 observamos que a Cidade do Ceara em Fortaleza situado na região Nordeste do país (Brasil) está com duas publicações de artigos; a Cidade de Vila Real em Portugal com um artigo; a Cidade de Campus em São Paulo com um artigo; a Cidade de São Paulo em São Paulo com um artigo; a Cidade de Niterói no Rio de Janeiro com um artigo; a Manaus em Amazonas com um artigo; a Cidade de Concórdia em Santa Catarina com um artigo; a Cidade de Vassouras em Rio de Janeiro com um artigo e a Cidade de Salvador na Bahia com um artigo publicado.

Cabe aqui ressaltar, que é na Região Sudeste que se encontra o maior número de Universidades do país e os maiores Centros acadêmico. Sendo assim o gráfico nos mostra que em todas as regiões do País e até mesmo no exterior e tema abordado estresse no

Grafico 01. Distribuição dos artigos de acordo com o ano de publicação selecionado no banco de informações da BVS no período de 2008 a 2017.

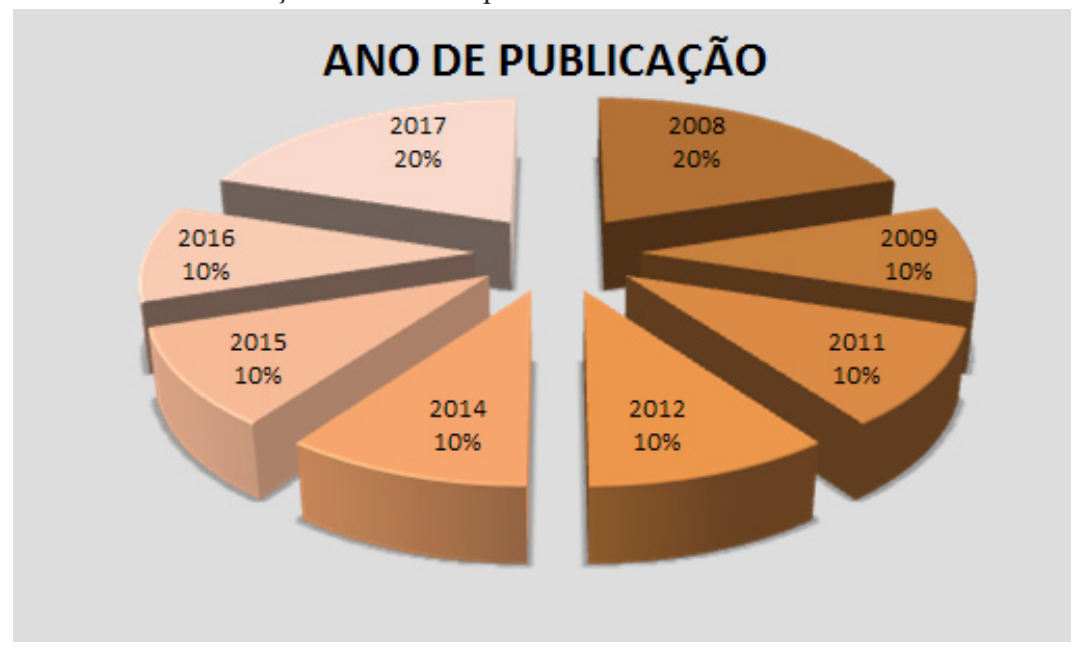

Gráfico 2. Distribuição dos artigos de acordo com o local de publicação da pesquisa.

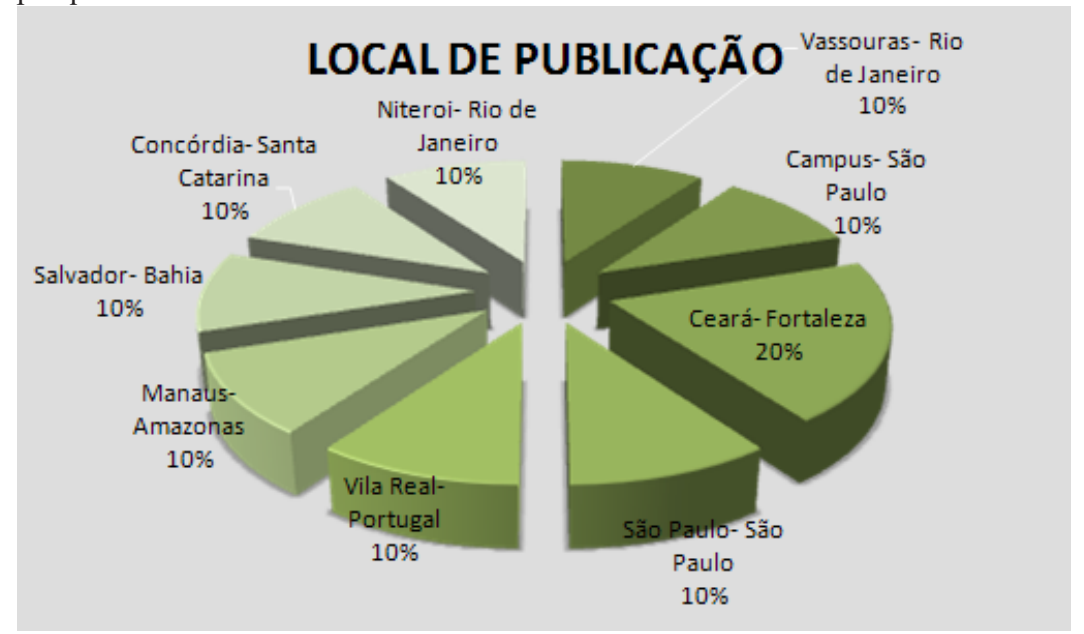




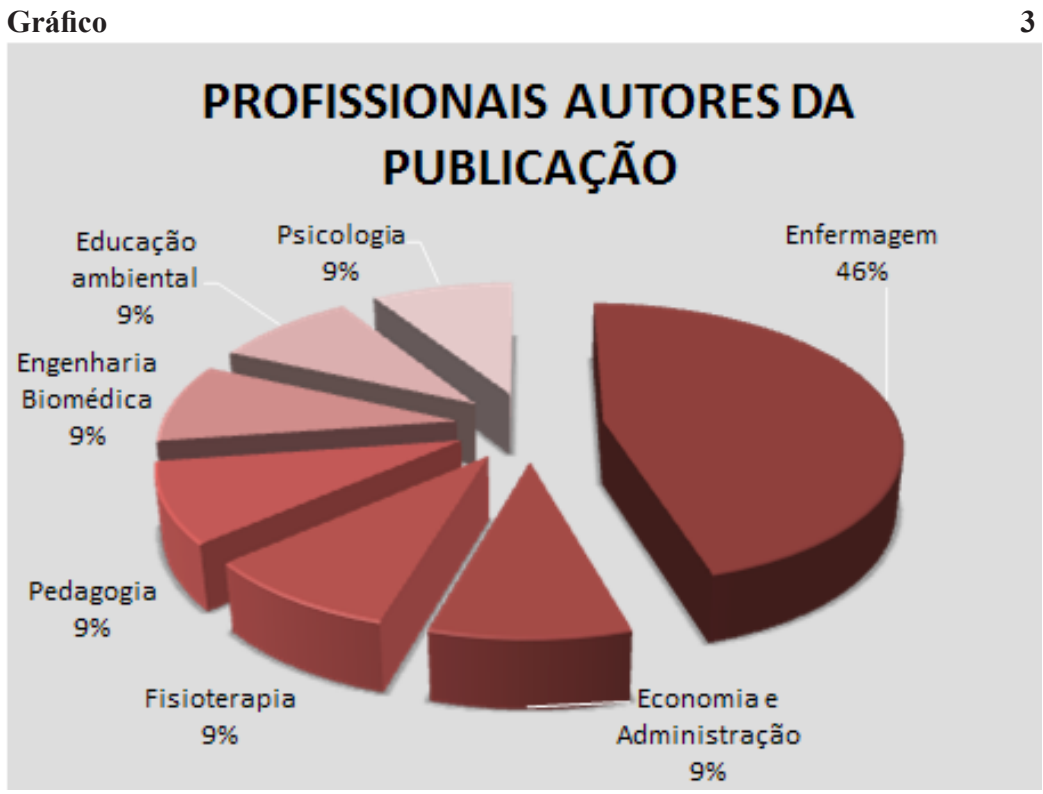

ambiente hospitalar é um assunto contemporâneo e de grande interesse para ser sempre discutido e pesquisado, ressaltando assim métodos para suas intervenções.

Analisando o gráfico de número três os protagonistas das publicações são da Enfermagem com 05 artigos, Economia e Administração com 01 artigo, Fisioterapia com 01 artigo, Pedagogia com 01 artigo, Engenharia Biomédica com 01 artigo, Educação ambiental e Psicologia com 01 artigo. Sendo assim é de muito valor lembrar que dentro de cada artigo científico os outros participantes não são das mesmas especialidades para bem exemplificar dos seguintes participantes na construção dos artigos os Psiquiatras e Biólogos fazem parte de alguns desses artigos. Nesse contexto podemos observar que todas as áreas das especialidades estudam o Estresse dentro de seus convívios. O Estresse é um fator que atinge a todas as pessoas, independente de sua área de profissão ou independe da situação do sujeito, seja ele em âmbito hospitalar, industrial, transito ou moradia. Em todos os lugares o efeito do estresse está presente.

Dos seguintes temas abordados pelos artigos científicos a Distribuição dos dados sócio demográfico dos enfermeiros com 3\%, Verificação de ruídos, luminosidade e temperatura com 3\%, Nível de estresse com $19 \%$, Acumulo de funções dos enfermeiros com

Gráfico 4. Dados analisados nos Artigos Científicos.

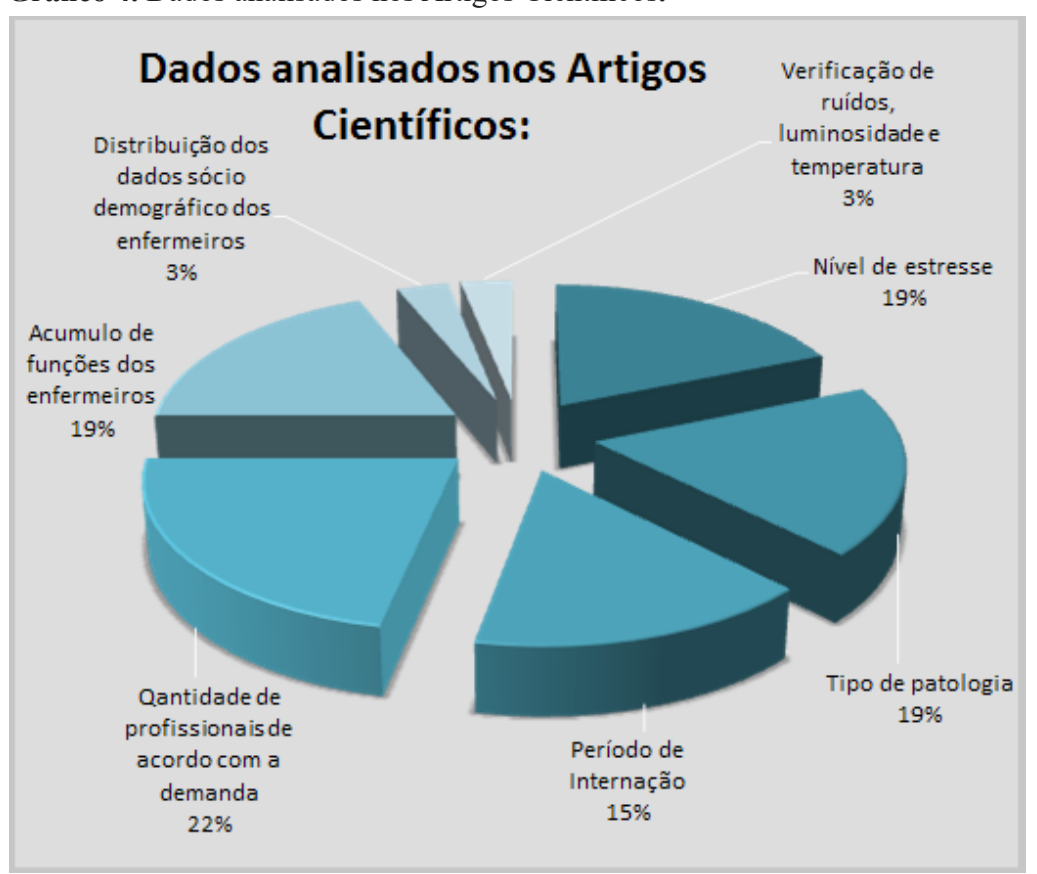


$19 \%$, Quantidade de profissionais de acordo com a demanda com $22 \%$, Período de internação com $15 \%$, Tipo de patologia com 19\%. Esses números nos mostram que as pesquisas científicas sempre irão abordar para o fator Estresse categorias parecidas em seus resultados.

\section{Discussão}

Os referenciais teóricos utilizados contribuíram para uma visão mais ampla sobre o estresse, em relação ao processo do adoecer e a hospitalização, possibilitando uma reflexão sobre os vários fatores relacionados e das teorias criadas como estratégias para padrão a serem seguidas na melhoria do estado de saúde do cliente, estratégias criadas por eles para aprimorar a assistência dos profissionais no enfrentamento dos clientes frente aos estressores.

Os principais fatores apresentados nesta pesquisa que contribuiu para o aparecimento do estresse são: distribuição dos dados sócio demográfico dos enfermeiros, Verificação de ruídos, luminosidade e temperatura, Nível de estresse, Acumulo de funções dos enfermeiros, Quantidade de profissionais de acordo com a demanda, Período de internação e Tipo de patologia.

Para certificação e concretização dos resultados que descrevem as intervenções de enfermagem no ambiente hospitalar e as definições do estresse e dos agentes estressores.

Identificaram a melhora de enfrentamento com uma intervenção de enfermagem definida como ajudar um cliente a se adaptar aos estressores. ${ }^{5}$

Desenvolveram escalas de eventos da vida que atribuem valores numéricos, denominados unidades de mudança de vida, aos eventos típicos da vida. ${ }^{6}$

Depois de um estressor, a pessoa reage consciente ou inconscientemente para lidar com a situação. Isso é denominado processo mediador. ${ }^{7}$

$O$ primeiro cientista a definir o termo stress no campo da saúde foi Hans Selye, um austríaco-canadense e médico endocrinologista, em 1956, que o descreveu como: o resultado inespecífico de qualquer demanda sobre o corpo, seja de efeito mental ou somático e estressor, todo agente ou demanda que evoca reação de estresse, seja de natureza física, mental ou emocional. ${ }^{8}$

O estresse é uma forma não específica de resposta com que o corpo se relaciona com certos agentes externos e internos. ${ }^{9}$

Definem o estresse como uma sobrecarga dos sistemas mental e físico de um indivíduo que pode ter muitas causas. ${ }^{10}$

Afirmam que o estresse pode ser definido como uma reação muito complexa, composta de alterações psicofisiológicas, que ocorrem quando o indivíduo é forçado a enfrentar situações que ultrapassem suas habilidades de enfrentamento. ${ }^{11}$
Observou que o estresse produzia reações de defesa e adaptação frente ao agente estressor e, com isso, descreveu a Síndrome Geral de Adaptação (SAG). ${ }^{12}$

Os dados discutidos neste trabalho possibilitam uma reflexão sobre a atuação das equipes que atuam no contexto hospitalar, sendo importante repensar sobre as formas de intervenções propostas, os quais envolvem o atendimento ao paciente e a equipe inserida no contexto hospitalar, e assim se buscar alternativas que permitam a obtenção de resultados positivos durante o processo de hospitalização, como também na pós-alta.

\section{Conclusão}

A partir do estudo foi possível observar que o Estresse no Ambiente Hospitalar baseado nas reflexões apresentadas, que nem sempre é um fator de desgaste emocional e físico, e sim, é um mecanismo natural de defesa do organismo. Recebem-se os estímulos internos e externos através do sistema nervoso e dependendo da forma como que esses estímulos são enfrentados poderão provocar alterações psicológicas e biológicas negativas, podendo levar ao estresse crônico. Nesse caso, ocorrem alterações fisiológicas que poderão desencadear vários tipos de doenças psicossomáticas.

Sabendo que da relevância que tem o estresse na vida dos profissionais da área da saúde e o seu efeito negativo é importante que sejam desenvolvidas medidas de enfrentamento com o objetivo de diminuir os problemas existentes no ambiente hospitalar, minimizar as dificuldades, dar apoio aos profissionais da área, proporcionar melhores condições de vida dentro e fora do ambiente hospitalar e assim, melhorar a qualidade da assistência prestada ao indivíduo.

Esse trabalho reafirma o papel e a importância da atuação do Enfermeiro no contexto hospitalar.

\section{Referências}

1. ABNT- associação brasileira de normas técnicas. NBR 14724: Informação e documentação: trabalhos acadêmicos: apresentação. Rio de Janeiro, 2002.

2. Tavares CMM. Como desenvolver experimentações estéticas para produção de dados na pesquisa sociopoética e abordagens afins? Revista Pró-UniverSUS. 2016 Jul./Dez.; 07 (3): 26-31.

3. Gil AC. Como elaborar projetos de pesquisa. 5. ed. São Paulo: Atlas, 2008 .

4. Tavares CMM. Prática criativa da enfermagem psiquiátrica: fatores intervenientes no seu desenvolvimento. Esc. Anna Nery. 2002;6(1):107118.

5. Brasil. Ministério da Saúde. Portaria GM/MS N ${ }^{\text {o } 336,19}$ de fevereiro de 2002, que regulamenta o funcionamento dos Centros de Atenção Psicossocial - CAPS, para atendimento público em saúde mental. Brasília, MS, 2002. Disponível em: http://bvsms.saude.gov.br/bvs/saudelegis/gm/2002/ prt0336_19_02_2002.html.

6. Mynaio MCS. Pesquisa social Teoria método e criatividade. $2^{\text {a }}$. Edição. Rio de *

7. Gauthier J. Sociopoética e Formação do Pesquisador Integral. Revista Psicologia, Diversidade e Saúde. 2016;4(1) 